\section{Women in science}

\section{In the hundred years since the creation of Interna- tional Women's Day, great strides have been made in gender equality, but recent analysis suggests the need for further changes to enhance the progression of women in science.}

This year marks the centenary of International Women's Day, originally created as part of the larger campaign for equal rights and opportunities for women. A century later, tremendous progress has been achieved in gender equality, access to political rights, education and employment for women around the world. A US Census Bureau report found that in 2009 women had pulled ahead of men in the attainment of higher education; women represented $58 \%$ of the surveyed population holding bachelor's degrees and advanced degrees. But despite these encouraging gains, women continue to earn considerably less than their male peers in the same educational category, including in STEM (science, technology, engineering and mathematics) occupations. For example, a 2008 study in the US found that although female biology scientists represented $53 \%$ of workers, their earnings only translate to $89 \%$ of the wages earned by men in these occupations. Two reports published in 2010, the AAAS/L'Oreal survey on roadblocks faced by women scientists to career progression and a report by the American Association of University Women (AAUW) analysing the under-representation of women in STEM professions, also offer a sobering counterpoint to the more optimistic news about women's gains in higher education in the US.

Of the 1,300 male and female respondents in the AAAS/L'Oreal survey, both groups cited the scarcity of jobs and funding resources as important barriers to career development, and a high proportion of both groups (83\%) knew of a colleague who had left science. Struggling with career-life balance and the challenges of having and raising children while maintaining a scientific career emerged as two key reasons causing women to leave science. Rather disturbingly, an astonishing $52 \%$ of women also reported having experienced gender-related bias, compared with $2 \%$ of male respondents. These findings regarding the particular challenges faced by women in STEM professions are echoed in the AAUW report.

So, what are the solutions for improving the retention rate and providing more equitable pay for women in science? Both reports found that mentorship and a supportive environment created by colleagues and peers can be an important factor in job satisfaction for women scientists. Recommendations in the AAUW report also emphasize a need for clear, transparent frameworks for evaluating performance and success, and an awareness of one's implicit bias. An earlier report by the AAUW on the gender pay gap suggested that some proportion of the pay gap may reflect the relative willingness of women and men to negotiate for competitive salaries and recommended better training for women scientists in negotiation strategies. The pay gap is often exacerbated for women who take career breaks to have a family. Flexible working practices, including in high-level positions, and other policies that aim to strike a better balance between career and family could potentially help reduce the pay gap and retain women in science.

\section{Focus on stem cells}

\section{This issue presents a series of specially commissioned articles that highlight exciting facets of stem cell research, including recent insights into the nature of pluripotency and how studying stem cells can increase our understanding of normal ageing and disease.}

Stem cells possess the unique capacity to self-renew and to activate one or several differentiation programs. Although cell replacement therapy is still far from being realized, recent research has been driven by the prospects that stem cells represent for regenerative medicine.

Since John Gurdon's discovery that the developmental clock of a differentiated nucleus could be reset to its pluripotent state, stem cells scientists have attempted to reprogramme somatic cells in a controlled manner. This was finally achieved by Shinya Yamanaka and colleagues in 2006 using a defined set of transcription factors. Since then, the pace of stem cell research has accelerated in the reprogramming field and in other areas essential for future clinical applications, including understanding how stem cell activity maintains organ homeostasis in mature tissues. To celebrate the blooming of this field, we are pleased to present Reviews and Perspective articles written by leading experts focusing on recent advances in stem cell research.

Sean Wu and Konrad Hochedlinger review recent progress and future challenges for researchers using patient-specific pluripotent cells obtained through reprogramming approaches. Studying these cells provides insights into the cellular and molecular basis of a range of diseases and offers opportunities for developing novel therapeutic tools. Several technical advances have increased our current understanding of stem cell physiology. The ability to maintain embryonic stem cells in vitro has meant that a systems approach can be applied to analyse the signalling and transcriptional networks responsible for pluripotency, and Huck-Hui Ng and Azim Surani examine the recent findings illustrating the dynamic nature of pluripotency. Understanding how the regenerative capacity of adult tissues is tapped to maintain homeostasis and activate repair is crucial for future therapeutic applications. A first step in this direction consists of identifying stem cells in their natural environment. Elaine Fuchs and Valerie Horsley consider advances in lineage-tracing technologies that have allowed the identification and functional characterization of stem cells within mammalian adult tissues. Finally, Thomas Rando and Leanne Jones discuss findings from both invertebrate and mammalian models that have led to an enhanced appreciation of age-related changes in stem cell function, including decreased self-renewal capacity and impaired differentiation, which ultimately result in reduced tissue maintenance and repair.

We hope that this Focus issue captures the excitement of stem cell research while also illustrating the numerous challenges to be met before these fundamental findings can be translated into clinical applications. 